\title{
A Theoretical Estimate of the Energy Barriers Between Stable Conformations of the Proline Dimer
}

\author{
C. M. VENKATACHALAM, B. J. PRICE, and S. KRIMM, \\ Harrison M. Randall Laboratory of Physics, Biophysics Research Division \\ and Macromolecular Research Center, University of Michigan, \\ Ann Arbor, Michigan 48104
}

\section{Synopsis}

Semi-empirical energy calculations for an internal Pro-Pro dimer are presented that take into account the nature of the flexibility of the proline ring due to its puckering. Calculations show that three stable conformations are available for the dimer: the cis $\left(\omega=0^{\circ}, \psi=160^{\circ}\right)$; the trans $\left(\omega=180^{\circ}, \psi=160^{\circ}\right.$, also referred to as trans $\left.{ }^{\prime}\right)$; and the $\operatorname{cis}^{\prime}\left(\omega=180^{\circ}, \psi=-40^{\circ}\right)$ conformations. The best conformational pathways between these stable conformations are determined. Calculations also show that the barrier for cis'-trans' conversion is of the same order of magnitude as that for cis-trans conversion.

\section{INTRODUCTION}

Several studies of the conformational energies of polyproline and of peptides containing prolines have been reported. ${ }^{1-9}$ Although a rigid pyrrolidine ring is employed in most of these studies, recent work ${ }^{10-14}$ indicates that the ring is quite flexible. A detailed calculation ${ }^{14}$ of the energies of the various puckered structures of the proline ring shows that the torsion angle $\varphi^{15}$ about the bond $\mathrm{N}-\mathrm{C}^{\alpha}$ can vary from $-35^{\circ}$ to $-85^{\circ}$ and that for a given value of $\varphi$, the ring can adopt different kinds of structures. Energy calculations ${ }^{13,14}$ show that there are three types of conformations that the Pro-Pro dimer may adopt: the trans $^{\prime}$. cis conformation, $\omega=0^{\circ}$, $\psi \approx 160^{\circ}$; the trans $^{\prime} \cdot$ trans conformation, $\omega=180^{\circ}, \psi \approx 160^{\circ}$; and the cis' trans conformation with $\omega=180^{\circ}, \psi \approx-40^{\circ}$. Here, trans $^{\prime}$ and cis' refer to the rotational states about the $\mathrm{C}^{\alpha}-\mathrm{C}$ bond while the unprimed terms refer as usual to the rotational states about the $\mathrm{C}-\mathrm{N}$ bond. Since a $c i s^{\prime}$. cis conformation is not available for the dimer, we shall refer to the trans' $\cdot c i s$, the cis' $\cdot$ trans, and the trans' $\cdot$ trans conformations, respectively, as the $c i s$, the $c i s^{\prime}$, and the trans (or the trans') conformations. While all energy calculations for the dimer show low-energy minima for the cis and trans' $^{\prime}$ conformations, earlier calculations ${ }^{1-3,16,17}$ using rigid proline rings gave a very high energy for the $c i s^{\prime}$ conformation. However, if backbone bond angles, the partial charges, and the ring geometry are suitably chosen, ${ }^{13,14}$ the energy of the cis $^{\prime}$ conformation becomes comparable to

(C) 1975 by John Wiley \& Sons, Inc. 
that of the trans' structure (see page 217 of Ref. 14 for a discussion of this point). Indeed, the existence of an occasional cis' conformation in polyproline in solution was suggested by the experimental studies of Steinberg et al. ${ }^{17}$ Also, theoretical calculations ${ }^{13}$ of the average end-to-end length of polyproline in solution are in agreement with the characteristic ratio of 14 obtained from viscometry by Mattice and Mandelkern ${ }^{18}$ if the energy of the cis' $^{\prime}$ conformation is only about $1.5 \mathrm{kcal} / \mathrm{mol}$ higher than that of the trans structure.

Examination of molecular models with planar imide residues shows that severe steric conflicts will produce a high-energy barrier for a transition from trans' to cis' $^{\prime}$ by $\psi$ rotation. Energy calculations ${ }^{14}$ show that this barrier is more than $100 \mathrm{kcal} / \mathrm{mol}$. On the other hand, the barrier for the cis-trans transition is only of the order of $20 \mathrm{kcal} / \mathrm{mol} .^{19}$ In view of this, the occurrence of an occasional cis conformation in polyproline seems a more attractive possibility than the occurrence of an occasional cis' residue, and it is known that a few percent of residues in the cis conformation could also account for the low characteristic ratio. However, the energy calculations presented in this paper using nonplanar residues and different types of puckered prolyl rings show that the cis'-trans' barrier is comparable to the cis-trans barrier. This result lends further support to the claim of Mattice et al. ${ }^{13}$ that the $c i s^{\prime}$ conformation is important in considering the average dimensions of polyproline in solution.

\section{PUCKERING OF THE PROLYL RING}

We have reported an extensive study ${ }^{14}$ of the prolyl ring puckering and its effect on the Pro-Pro internal dipeptide. We shall briefly summarize the relevant details. Consider an isolated prolyl ring shown in Figure 1. All bond lengths are held constant. The bond angle $\tau^{\alpha}$ at $\mathrm{C}^{\alpha}$ and the bond angles $\tau^{\mathrm{N}}, \tau^{\mathrm{N}^{\prime}}$, and $\tau^{\mathrm{N}^{\prime \prime}}$ at $\mathrm{N}$ are also held constant. Assuming $\mathrm{C}^{\alpha}$ to be tetrahedral and $\mathrm{N}$ to be planar trigonal, the torsion angle $\theta$ associated with the atoms $\mathrm{C}^{\delta}, \mathrm{N}, \mathrm{C}^{\alpha}$, and $\mathrm{C}^{\beta}$ determines the position of all atoms shown except $\mathrm{C}^{\gamma}$. (There is a linear relation between $\theta$ and the torsion angle $\varphi$.)

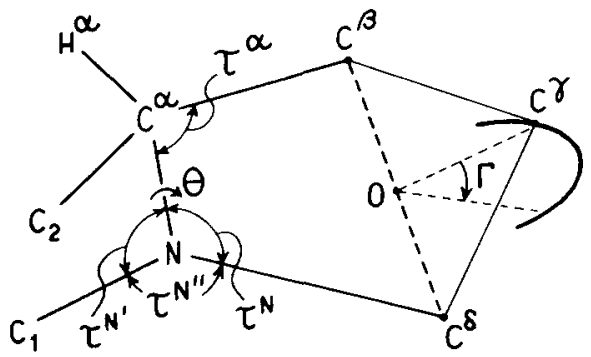

Fig. 1. The geometry of the proline ring. $\mathrm{O}$ is the center of the $\mathbf{\Gamma}$ circle and lies on the line $\mathrm{C}^{\delta_{-}--} \mathrm{C}^{\beta}$. The dihedral angle $\theta$ is associated with the atoms $\mathrm{C}^{\delta}-\mathrm{N}-\mathrm{C}^{\alpha}-\mathrm{C}_{\beta}$ and is related to $\varphi$. The methylene hydrogens are not shown here, though they have been included in the calculations. 


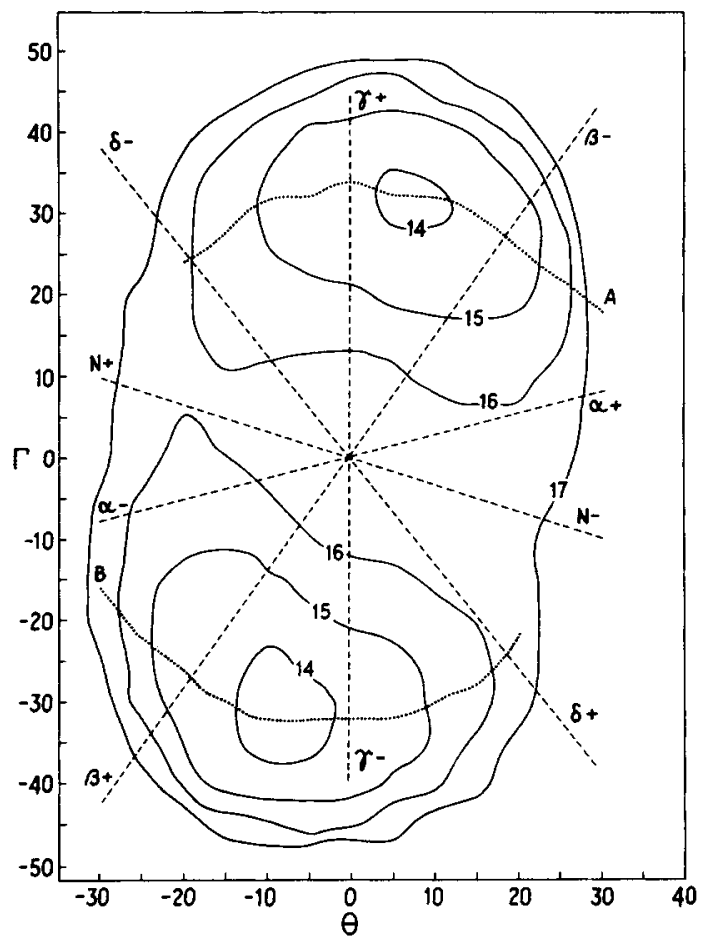

Fig. 2. $\Gamma-\theta$ conformational energy plot for an isolated proline ring shown in Fig. 1. Every point on this plot represents a specific prolyl ring geometry. The dashed lines represent single-atom-puckered geometries. See text for the definition of $\Gamma=0^{\circ}$. $\theta=\varphi+61.3^{\circ}$.

Since bond lengths are held fixed, the atom $\mathrm{C}^{\gamma}$ has to lie on a circle with its center $\mathrm{O}$ lying on the line $\mathrm{C}^{\beta}---\mathrm{C}^{\delta}$. Various positions of $\mathrm{C}^{\gamma}$ on this circle may be obtained by rotating $\mathrm{O}---\mathrm{C}^{\gamma}$ about the line $\mathrm{C}^{\beta}-\mathrm{C}^{\delta}$ through an angle $\Gamma$. Thus, the angles $\Gamma$ and $\theta$ completely determine the conformation of the ring. The hydrogens on $\mathrm{C}^{\beta}, \mathrm{C}^{\gamma}$, and $\mathrm{C}^{\delta}$ are fixed such that the plane $\mathrm{HCH}$ bisects the appropriate $\mathrm{C}-\mathrm{C}-\mathrm{C}$ or $\mathrm{C}-\mathrm{C}-\mathrm{N}$ angle. The bond lengths employed are $l\left(\mathrm{C}^{\alpha}-\mathrm{C}^{\beta}\right)=1.53 \AA, l\left(\mathrm{C}^{\beta}-\mathrm{C}^{\gamma}\right)=1.50 \AA$, $l\left(\mathrm{C}^{\gamma}-\mathrm{C}^{\delta}\right)=1.52 \AA, l\left(\mathrm{~N}-\mathrm{C}^{\delta}\right)=1.48 \AA, l\left(\mathrm{~N}-\mathrm{C}^{\alpha}\right)=1.47 \AA$, and the bond angles are $\tau^{\alpha}=103^{\circ}, \tau^{\mathrm{N}}=113^{\circ}, \tau^{\mathrm{N}^{\prime}}=121^{\circ}, \tau^{\mathrm{N}^{\prime \prime}}=126^{\circ}$, and $\tau\left(\mathrm{NC}^{\alpha} \mathrm{C}\right)=$ $110.9^{\circ}$. With these parameters, the exact relation between $\varphi$ and $\theta$ is found to be $\varphi=\theta-61.3^{\circ}$.

The total conformational energy of the isolated proline ring is computed as a sum of van der Waals and electrostatic interactions, torsional potentials, and bond-angle deformation energies ${ }^{14}$ and the dependence of this ring "self energy" on $\Gamma$ and $\theta$ is depicted in Figure 2. Every point on the $\Gamma-\theta$ plot represents a specific ring geometry. For each $\theta, \Gamma=0^{\circ}$ corresponds to the structure in which the atom $\mathrm{C}^{\gamma}$ lies in the plane that bisects the angle between the planes $\mathrm{C}^{\delta}-\mathrm{N}-\mathrm{C}^{\alpha}$ and $\mathrm{N}-\mathrm{C}^{\alpha}-\mathrm{C}^{\beta}$. (It is found that the ring torsion angles $\chi^{\mathrm{N} \delta}$ and $\chi^{\alpha \beta}$ are roughly equal at $\Gamma=0^{\circ}$.) 


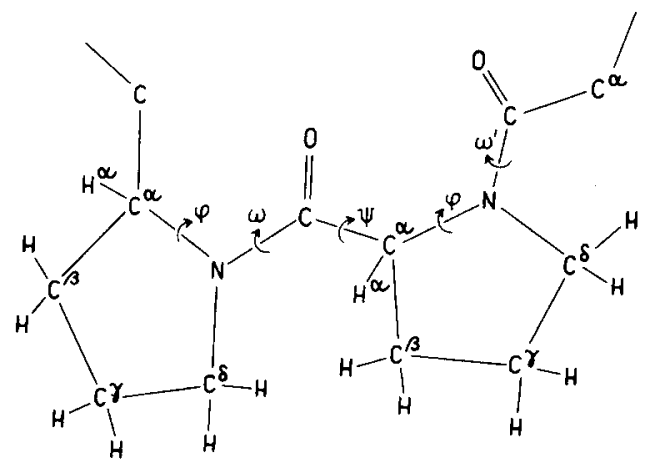

Fig. 3. The Pro-Pro internal dipeptide.

The proline ring is perfectly $X$-puckered if the torsion angle about the bond opposite to atom $X$ is zero. $X^{-}$puckering means that the atom $X$ lies on the same side of the ring as the carbonyl carbon $\mathrm{C}_{2}$ (Figure 1 ); if the atom $X$ is on the other side of the ring, it is $X^{+}$puckered. ${ }^{20}$ The straight dashed lines in the $\mathrm{\Gamma}-\theta$ plot represent $\mathrm{C}^{\gamma}$ positions for which the ring is perfectly single-atom-puckered. It is also convenient to classify ring geometries into two types ${ }^{10} \mathrm{~A}$ and $\mathrm{B}$ based on the sign of the torsion angle $\chi^{\delta \gamma}$. The ring is of type $\mathrm{A}$ if $\chi^{\delta \gamma}$ is negative and it is of type $\mathrm{B}$ for positive $\chi^{\delta \gamma}$. Thus, all the points above the $\alpha$ line in the $\Gamma-\theta$ plot represent type-A ring structures while those lying below the $\alpha$ line are of type B. For each value of $\theta$, in general, the ring energy is a minimum for two values of $\Gamma$ corresponding to type-A and B structures. The dotted lines marked A and B show these minimum-cnergy structures for each $\theta$. The global encrgy minimum occurs for $\theta=-10^{\circ}$ and the energy of the planar ring $\left(\Gamma=\theta=0^{\circ}\right)$ is $3.2 \mathrm{kcal} / \mathrm{mol}$ higher than that of the global energy minimum structure. Also, the ring energy $\Gamma-\theta$ plot shows that the ring is essentially $\gamma$-puckered for values of $\theta$ close to $0^{\circ}$ (i.e., for $\varphi \sim-60^{\circ}$ ) and the lowest energy ring structure is $\beta$-puckered when $|\theta| \sim 20^{\circ}$ (i.e., for $\varphi \sim-40^{\circ}$ and for $\varphi \sim$ $\left.-80^{\circ}\right)$.

\section{ENERGY OF THE PRO-PRO INTERNAL DIPEPTIDE}

The chemical structure of the Pro-Pro internal dipeptide is depicted in Figure 3. The potential functions used in calculating the dimer energies are essentially those employed earlicr. ${ }^{14}$ The partial charges on the atoms are from Goodman et al. ${ }^{21} \psi$ and $\omega$ were varied from $-180^{\circ}$ to $180^{\circ}$ in increments of $20^{\circ}$. $\theta$ was varied from $-25^{\circ}$ to $25^{\circ}$ in intervals of $5^{\circ}$. The intrinsic torsional potential for the $\omega$ rotation was taken as

$$
V_{\omega}=1 / 2 V_{0}(1-\cos 2 \omega)
$$

where $V_{0}=20 \mathrm{kcal} / \mathrm{mol}$. (While varying $\omega$, we have kept the partial charges constant. There will undoubtedly be some variation of the eharges with $w$. On the other hand, Yan and co-workers ${ }^{22}$ found from CNDO/2 


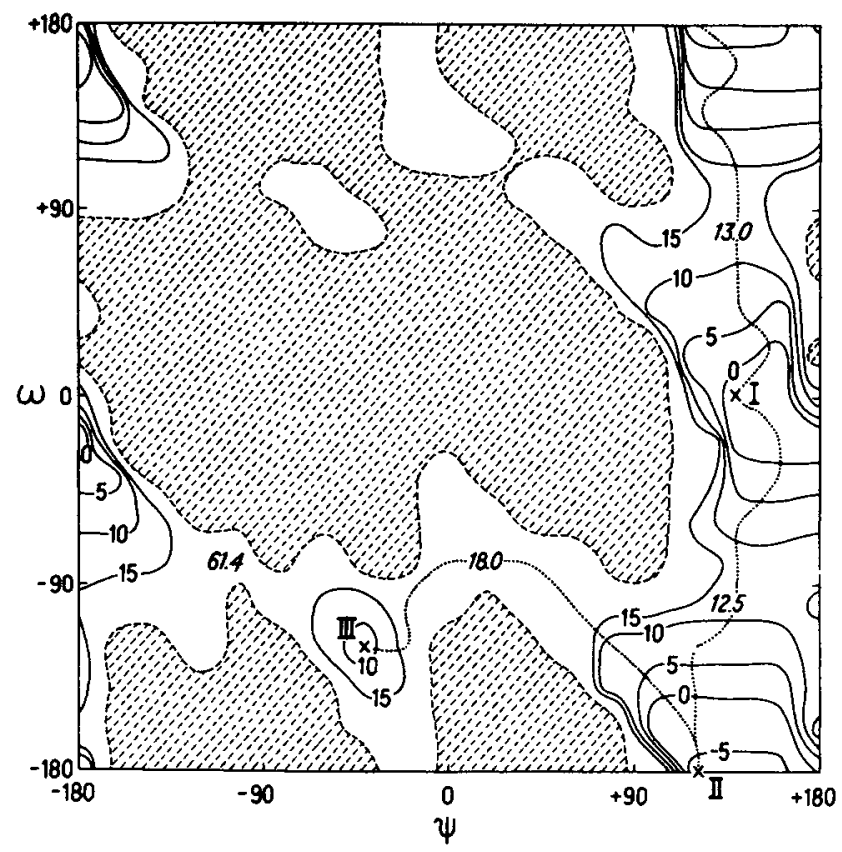

(a)

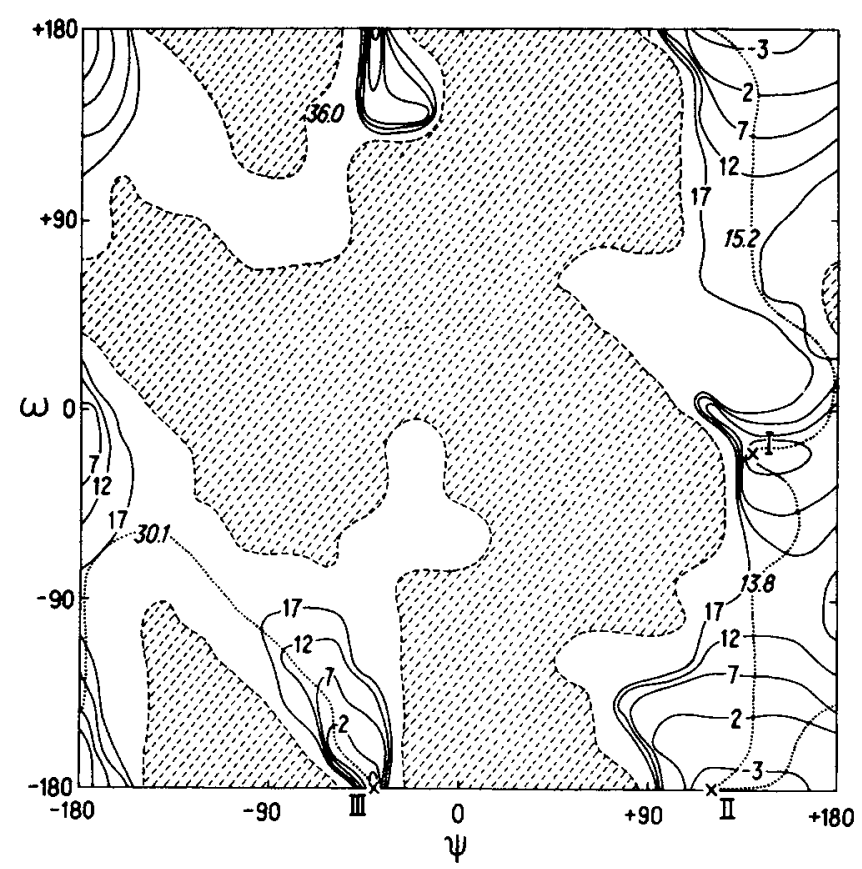

(b)

Fig. 4 (continued) 


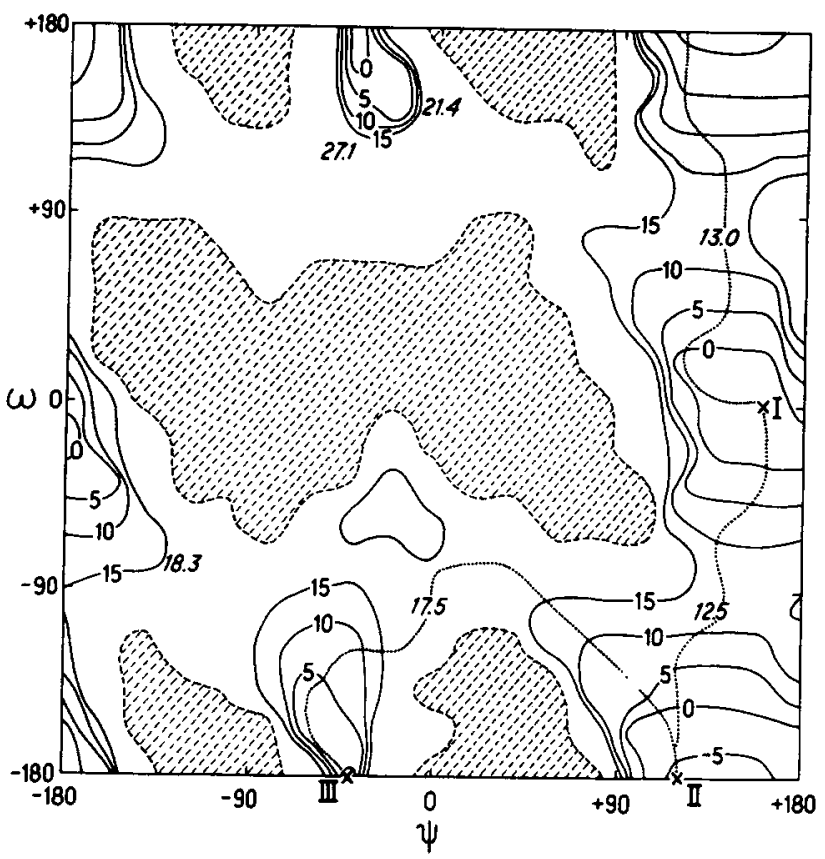

(c)

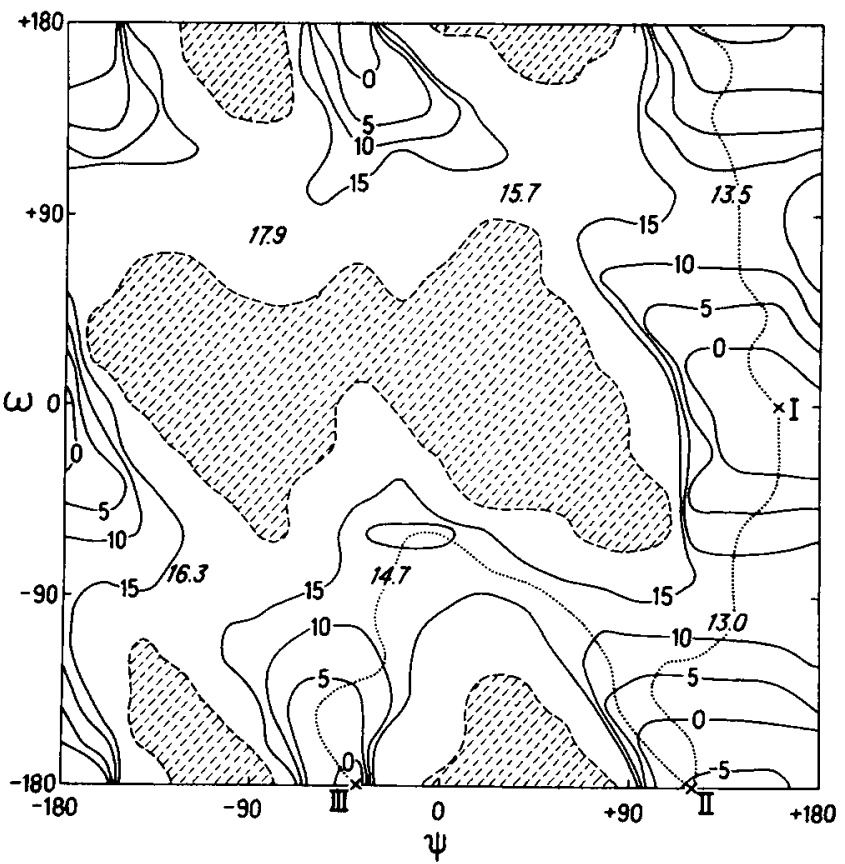

(d)

Fig. 4 (continued) 


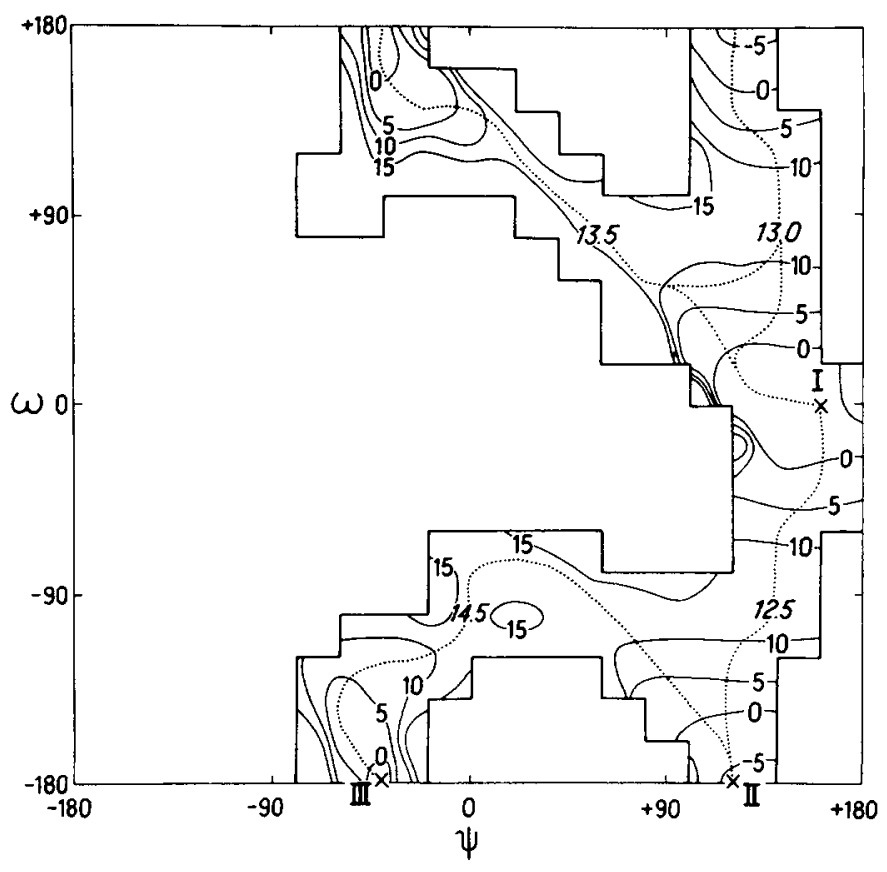

(e)

Fig. 4. The $\omega-\psi$ energy maps for the Pro-Pro internal dipeptide. The contours are in intervals of $5 \mathrm{kcal} / \mathrm{mol}$. The regions with energy greater than $75 \mathrm{kcal} / \mathrm{mol}$ have been hatched. The energy minima are marked by crosses. Regions marked I, II, III are, respectively, the $c i s$, trans $^{\prime}$, and $c i s^{\prime}$ conformations. The pathways between the energy minima have been shown by dotted lines and the energy at the barrier along each path is marked in italics. (a) $\theta=-10^{\circ}\left(\varphi \approx-71^{\circ}\right)$. (b) $\theta=10^{\circ}\left(\varphi \approx-51^{\circ}\right)$. (c) For each $\omega, \psi$, the best value of $\theta$ giving the lowest dimer energy is taken. (d) Same as in (c), but the bond length $l(\mathrm{C}-\mathrm{N})$ is linearly increased from 1.32 to $1.47 \AA$ as $\omega$ goes from $0^{\circ}$ (or $180^{\circ}$ ) to $\pm 90^{\circ}$. (e) Same as in (c), but the two rings have been independently puckered so that $\theta_{1}$ is not necessarily equal to $\theta_{2}$. Calculations were performed only for the value of $\omega, \psi$ shown in this figure.

calculations only small variation of charges with $\omega$ for $N$-methylacetamide. It therefore seems that nonvariation of partial charges may be a fair approximation.) The following values of the geometrical parameters were used: $l(\mathrm{C}-\mathrm{O})=1.24 \AA, l(\mathrm{C}-\mathrm{N})=1.32 \AA, \tau\left(\mathrm{C}^{\alpha}-\mathrm{C}-\mathrm{O}\right)=122^{\circ}, \tau\left(\mathrm{C}^{\alpha}-\right.$ $\mathrm{C}-\mathrm{N})=119^{\circ}$. For each $\theta$, all five single-atom-puckered structures as well as the types-A and B minimum-energy ring structures were considered for the prolyl ring.

Figure 4 a shows the energy variation with $\omega$ and $\psi$, if $\theta$ is held constant at $-10^{\circ}\left(\varphi \approx-71^{\circ}\right)$, both the rings having identical puckering. The energy contours are in intervals of $5 \mathrm{kcal} / \mathrm{mol}$. The regions where the dimer energy is greater than $75 \mathrm{kcal} / \mathrm{mol}$ have been hatched. The energy minima are marked by crosses and are indicated by numerals I, II, and III roughly corresponding to the cis, trans', and cis' conformations, respec- 
tively. The best conformational pathways between minima are indicated by dotted lines. Along each path, the energy at the barrier is marked in italics. The values of the energy at the minima are given in Table I. Table II lists the energy and conformation at the barriers along the best conformational pathways between the minima. The global energy minimum is for the trans' conformation at region II. The energy at the trans-cis barrier is $12.5 \mathrm{kcal} / \mathrm{mol}$ along the best pathway. One of the consequences of restricting $\theta$ to $-10^{\circ}$ is that the energy and the location of the $c i s^{\prime}$ conformation is quite abnormal: the energy is about $14.2 \mathrm{kcal} / \mathrm{mol}$ greater than that of the trans' structure and the cis' structure has a highly nonplanar imide bond. A pathway for trans'-cis' conversion does exist with a barrier energy (by that we mean in this paper, the energy at the barrier rather than the barrier height, which normally refers to the difference between the energy at the barrier and that at a given minimum) of $18.0 \mathrm{kcal} / \mathrm{mol}$ and the conversion involves an $\omega$ rotation of about $100^{\circ}$. This explains the difficulty in converting from a trans' to a $\mathrm{cis}^{\prime}$ structure using molecular space-filling models that employ planar imide bonds.

A similar calculation with the value of $\theta$ restricted to $10^{\circ}\left(\varphi \approx-51^{\circ}\right)$, while keeping the puckering of both the rings identical, yields the energy map shown in Figure $4 \mathrm{~b}$. The trans-cis barrier is only slightly affected by this change in $\theta$, but the $\mathrm{cis}^{\prime}$ conformation now emerges as a distinct possibility with an energy of only about $0.8 \mathrm{kcal} / \mathrm{mol}$ higher than that of the trans' conformation, and the minimum occurs for a planar imide bond; but the pathway for trans'-cis', seen in Figure 4a, is now blocked. Another pathway has opened up now but the barrier is high.

These energy maps in Figure $4 a$ and b clearly demonstrate the importance of the ring flexibility in estimating the barrier energies. If the restriction of $\theta$ to a specific value is relaxed, once again with both the rings having identical puckering, the energy map in Figure $4 \mathrm{c}$ is obtained. Here, for each $\omega, \psi$, the best value of $\theta$ giving the lowest dimer energy, is employed. The map shows a marked increase in the steric freedom in the $\omega$ and $\psi$ rotation especially in region III. Once again, the cis-trans barrier is only slightly affected; but now, not only does the $c i s^{\prime}$ conformation have a low energy but also the barrier energy for trans'-cis' is $17.5 \mathrm{kcal} / \mathrm{mol}$, only 5 $\mathrm{kcal} / \mathrm{mol}$ higher than that for trans-cis conversion.

The cis' conformation is a rather compact structure and the energy in region III is sensitively dependent on the backbone bond angles, the ring puckering, and the potential functions employed, especially the partial charges on the atoms. ${ }^{13,14}$ (The backbone bond angles used in these calculations are those that are conducive to the stability of the $\mathrm{cis}^{\prime}$ conformation.) In view of this situation, it is interesting to study the effect of varying any relevant parameter involved in this problem. We give here one such example: as the value of $\omega$ is varied from the planar value of $0^{\circ}$ to the extreme nonplanar value of $90^{\circ}$ (or from $-180^{\circ}$ to $-90^{\circ}$ ), it is reasonable to assume that the bond length $\mathrm{C}-\mathrm{N}$ should be varied from its standard partial double-bond value of $1.32 \AA$ to the single-bond value of $1.47 \AA$. 


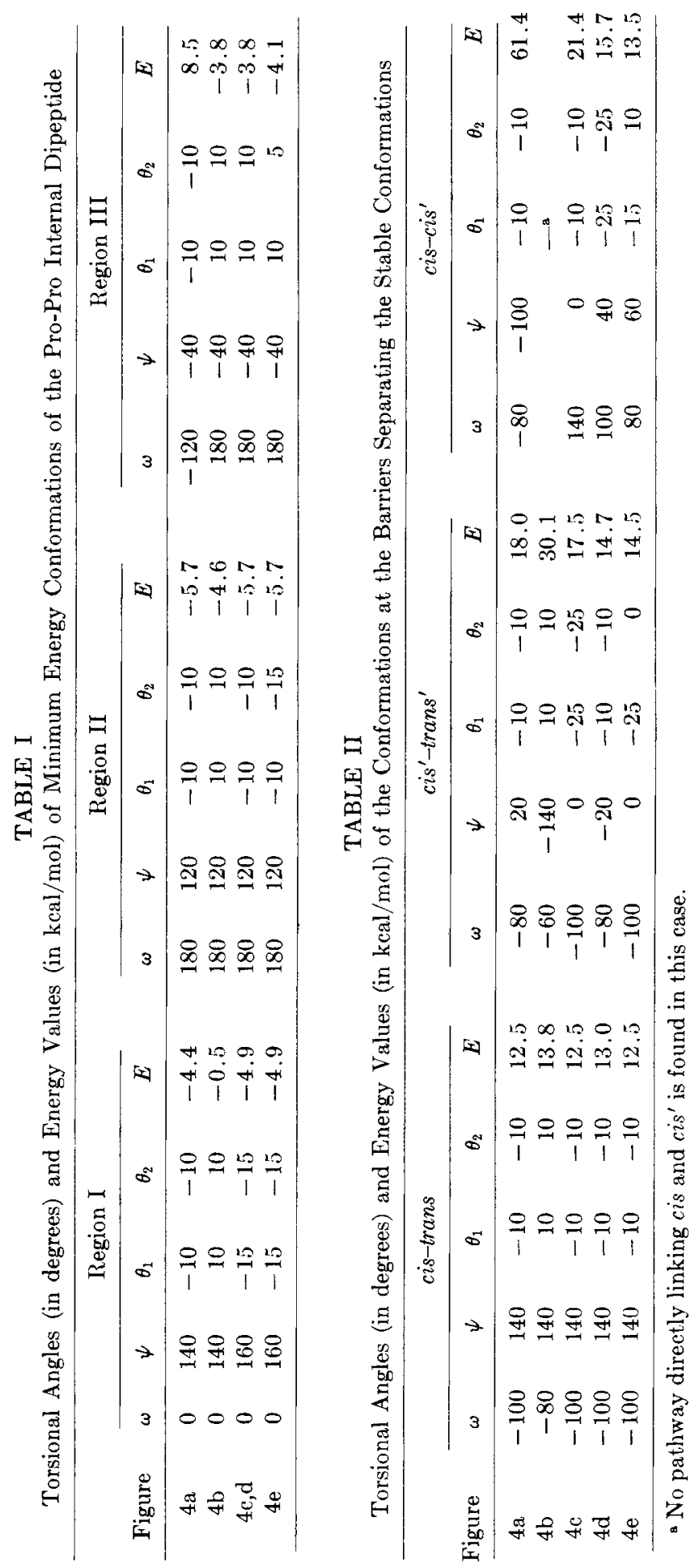


[In fact, at $\omega=90^{\circ}$ the double-bond character of the $\mathrm{C}-\mathrm{N}$ bond may be expected to be completely removed, in which case $l(\mathrm{C}-\mathrm{N})$ will stretch to the single-bond value of $1.47 \AA$, the energy required for this increase in bond length being already accounted for in the energy required for breaking the partial double bond (viz., about $20 \mathrm{kcal} / \mathrm{mol}$ ).] Accordingly, we have varied $l(\mathrm{C}-\mathrm{N})$ linearly from 1.32 to $1.47 \AA$ as the imide bond is taken from a planar conformation to its extreme nonplanarity. Figure $4 \mathrm{~d}$ shows the resulting energy map. The map shows, in general, increased flexibility for the dimer. In particular, the cis'-trans' barrier energy drops to a low value of $14.7 \mathrm{kcal} / \mathrm{mol}$, comparable to that for cis-trans. Also, the best pathway for $c i s^{\prime}-c i s$ has a low barricr energy of $15.7 \mathrm{kcal} / \mathrm{mol}$. We present this map here primarily to illustrate the point that variation of the geometrical parameters tends to improve the conformational pathway leading to the cis' region.

Up to this point, we have kept the puckerings of both the rings identical. Obviously, it is desirable to see the effect of relaxing this restriction. Accordingly, for cach $\omega, \psi$ the rings were independently puckered by varying $\theta_{1}$ and $\theta_{2}$ from $-30^{\circ}$ to $+30^{\circ}$ in $5^{\circ}$ increments. However, the $\mathrm{C}-\mathrm{N}$ length was kept constant at $1.32 \AA$. (In this case, the variation of the $\mathrm{C}-\mathrm{N}^{\top}$ length was found to produce only small changes in the energies.) The lowest energy for each $\omega, \psi$ has been plotted in Figure 4c. In order to keep the computing time reasonably low, we have restricted this calculation to the regions of interest here. The energies of the stable conformations are not significantly altered. There is further reduction in the barrier energies due to increased flexibility. In particular, the cis-cis' barrier at $\omega=80^{\circ}$, $\psi=60^{\circ}$ is reduced to $13.5 \mathrm{kcal} / \mathrm{mol}$, while the $c i s^{\prime}-t^{\circ} a n s^{\prime}$ barrier is reduced to $14.5 \mathrm{kcal} / \mathrm{mol}$. Thus, the barriers for the transitions cis-trans, cis'trans, and cis-cis' are of the same order of magnitude.

\section{DISCUSSION}

The energy maps developed here for the internal Pro-Pro dimer support the claim of Mattice et al. ${ }^{13}$ that the cis' conformation is one of the possible stable structures for the dimer. The calculations also indicate that pathways do exist to convert the cis or trans' conformation to the cis' conformation and viee versa. Further, the barrier height for trans'-cis' conversion is comparable to that for the trans-cis conversion, in agreement with the experimental studies of Stcinberg et al. ${ }^{17}$ who have estimated the barricr height for the trans'-cis' conversion to be $20.6 \mathrm{kcal} / \mathrm{mol}$ for a dilute aqueous solution of polyproline in $12 \mathrm{M} \mathrm{LiBr}$. Also, the trans'-cis' conversion involves an $\omega$ rotation of about $100^{\circ}$. This particular fact may be important in understanding the mechanism of the trans'-cis' transition in solution. It is important to note that the calculations presented here are for a dimer depicted in Figure 3. Thus the minimum energies and the energy barriers in Figure 4 are strictly applicable only for such a dimer. In the polymer, however, interactions between non-neighboring units will be 
quite important especially when one of the imide bonds takes up a cis conformation. ${ }^{6.9}$ Model building shows that this is also the case when there is a $\mathrm{cis}^{\prime}$ conformation. Thus the barrier heights for conformational transitions in polyproline will be affected by other factors (depending on the actual mechanism of the transition) in addition to those considered in this paper. Thus, caution should be exercised when comparing the barrier heights for the dimer and those experimentally measured for polyproline.

The fact that the cis'-trans' $^{\prime}$ barrier is of the same order as that for the cis-trans barrier is significant in the nmr analysis of polypeptides containing proline. The implication of our calculation is that the three conformations of the Pro-Pro dimer should lead to separate nuclear magnetic resonances at room temperature. Thus, this situation may be quite different from the case of an isolated proline for which the cis'-trans' barrier height has been calculated by Tonelli ${ }^{5}$ to be less than $10 \mathrm{kcal} / \mathrm{mol}$. Of course, these relative numerical values may change when explicit solvent interactions are incorporated. ${ }^{23}$ On the other hand, only two separate resonances have been observed in the nmr of polyproline studied by Dorman and co-workers. ${ }^{24}$ It may also be noted that the energy calculations presented here show that the amount of $c i s^{\prime}$ conformation present in polyproline is not likely to be more than about $5 \%$.

The calculations clearly demonstrate the role of the puckering of the pyrrolidine ring in such structural transformations. The exact nature of the puckering of the ring is important at least in the type of conformations considered here. For other molecules containing proline, the details of ring puckering may or may not be important in energy calculations. In each specific case, the importance of considering ring puckering must be evaluated by model building or energy calculations.

Calculations of this nature are obviously approximate and they do leave room for further improvement. In particular, the nitrogen has been kept planar trigonal. The assumption of the $s p^{3}$ quaternary state for the imide nitrogen will somewhat modify the conclusions. ${ }^{25}$ Recent CNDO/2 calculations by Ramachandran et al. ${ }^{26}$ indicate that the stable conformation of the peptide unit in $N$-methylacetamide is likely to be nonplanar with pyramidal nitrogen. In view of this, there is obviously a need for studying the possibility of pyramidal nitrogen in proline.

This research was supported in part by National Science Foundation Grant GB-15682. The authors thank Sidney Meier for assistance with some of the calculations.

\section{References}

1. DeSantis, P., Giglio, E., Liquori, A. M. \& Ripamonti, A. (1965) Nature 206, 456-458.

2. Schimmel, P. R. \& Flory, P. J. (1967) Proc. Nat. Acad. Sci. U.S. 58, 52-59.

3. Schimmel, P. R. \& Flory; P. J. (1968) J. Mol. Biol. 34, 105-120.

4. Hopfinger, A. J. \& Walton, A. G. (1969) J. Macromol. Sci. Phys., Sec. B 3, 171-193.

5. Tonelli, A. E. (1973) J. Amer. Chem. Soc. 95, 5946-5948.

6. Tonelli, A. E. (1971) J. Amer. Chem. Soc. 93, 7153-7155.

7. Maigret, B., Perahia, D. \& Pullman, B. (1970) J. Theor. Biol. 29, 275-291. 
8. Pullman, B.\& Pullman, A. (1974) Advan. Protein Chem. 28, 347-526.

9. Tanaka, S. \& Scheraga, H. A. (1974) Macromolecules 7, 698-705.

10. Ramachandran, G. N., Lakshminarayanan, A. V., Balasubramanian, R. \& Tegoni, G. (1970) Biochim. Biophys. Acta 221, 16.̌-181.

11. Nishikawa, K. \& Ooi, T. (1971) Progr. Theor. Phys. 46, 670-672.

12. Nishikawa, K. \& Ooi, T. (1972) Bull. Inst. Chem. Res., Kyoto Univ. 50, 94-106.

13. Mattice, W. L., Nishikawa, K. \& Ooi, T. (1973) Macromolecules 6, 443-446.

14. Venkatachalam, C. M., Price, B. J. \& Krimm, S. (1974) Macromolecules 7, 212-220.

15. IUPAC-IUB Abbreviations and Symbols for the Description of the Conformation of Polypeptide Chains (1970) J. Mol. Biol. 52, 1-17.

16. Go, N. \& Scheraga, H. A. (1970) Macromolecules 3, 188-194.

17. Steinberg, I. Z., Harrington, W. F., Berger, A., Sela, M. \& Katchalski, E. (1960) J. Amer. Chem. Soc. 82, 5263 5279.

18. Mattice, W. L. \& Mandelkern, L. (1971) J. Amer. Chem. Soc. 93, 1769-1777.

19. Phillips, W. D. (1955) J. Chem. Phys. 23, 1363-1364.

20. Torchia, D. A. (1971) Macromolecules 4, 440-442.

21. Goodman, M., Chen, V., Benedetti, E., Pedone, C. \& Corradini, P. (1972) Biopolymers 11, 1779-1787.

22. Yan, J., Momany, F., Hoffmann, R. \& Scheraga, H. A. (1970) J. Phys. Chem. 74, 420-433.

23. Krimm, S. \& Venkatachalam, C. M. (1971) Proc. Nat. Acad. Sci. U.S. 68, 24682471 .

24. Dorman, D. E., Torchia, D. A. \& Bovey, F. A. (1973) Macromolecules 6, 80-82.

25. Hopfinger, A. J. (1973) Conformational Properties of Macromolecules, Academic, New York p. 187.

26. Ramachandran, G. N., Lakshminarayanan, A. V. \& Kolaskar, A. S. (1973) Biochim. Biophys. Acta 303, 8-13.

Received June 7, 1974

Accepted December 26, 1974 